Rev. Biol. Trop. 52(1): 157-162, 2004

www.ucr.ac.cr www.ots.ac.cr www.ots.duke.edu

\title{
Composición, crecimiento e índice de condición de una población de Poecilia reticulata (Pisces: Poeciliidae), en un estanque en Heredia, Costa Rica
}

\author{
Mario Urriola Hernández, Jorge Cabrera Peña \& Maurizio Protti Quesada \\ Laboratorio de Recursos Naturales y Vida Silvestre (LARNAVISI), Escuela de Ciencias Biológicas, Universidad Nacional, \\ Heredia 86-3000, Costa Rica; jcabrer@samara.una.ac.cr, mprotti@una.ac.cr \\ Recibido 25-V-2001. Corregido 24-XI-2003. Aceptado 28-XI-2003.
}

\begin{abstract}
A pond population of Poecilia reticulata was studied in Santo Domingo, Heredia, Costa Rica, between September and November of 1998. The sex ratio was 1:0.49 (males:females). The mean total length was $34.43 \pm 7.26 \mathrm{~mm}$ for females, $23.50 \pm 2.24 \mathrm{~mm}$ for males and $12.27 \pm 3.41 \mathrm{~mm}$ for juveniles. The mean total weight was $0.69 \pm 0.48 \mathrm{~g}$ for females; and $0.16 \pm 0.05 \mathrm{~g}$ for males and $0.026 \pm 0.027 \mathrm{~g}$ for juveniles. The total length-weight relationship for the total population was $\mathrm{P}=6 \times 10^{-5} \mathrm{Lt}^{3.3272}\left(\mathrm{r}^{2}=0.9613\right)$. The condition index equation was $\mathrm{K}=25.755 \mathrm{e}^{0.004 \mathrm{Lt}}\left(\mathrm{r}^{2}=0.8925\right)$ for females and $\mathrm{K}=26.767 \mathrm{e}^{0.003 \mathrm{Lt}}\left(\mathrm{r}^{2}=0.907\right)$ for males. The mean condition index was $31.29 \pm 0.55 \%$ for females and $28.52 \pm 0.19 \%$ for males. Both sexes reached the sexual maturity when the males and females overcame the $20.00 \mathrm{~mm}$ and $23.5 \mathrm{~mm}$ of Lt respectively.
\end{abstract}

Key words: Poecilia reticulata, length, weight, condition index, Poeciliidae, Costa Rica.

Bussing (1998) mencionó, que entre las especies de peces exóticos introducidos en Costa Rica, están Poecilia reticulata (Peters, 1859), que se encuentra en ríos y acequias del Valle Central y Xiphophorus variatus (Meek, 1904) que se le localiza en varios ríos de la cuenca del río Reventazón. Lody (1978) señaló que los poecílidos se han introducido en muchas localidades tropicales, ya que son útiles para el control de plagas hematófagas y transmisoras de enfermedades al hombre, principalmente de mosquitos de los géneros Anopheles y Aedes que, según Vargas y Vargas (2003) constituyen un problema importante en cuerpos de aguas naturales y artificiales. $P$. reticulata fue introducido a Costa Rica desde Venezuela y Trinidad con este fin, por ser una especie que soporta algún grado de contaminación del agua (Bussing 1998).

$P$. reticulata, conocido comúnmente como gupi, presenta un marcado dimorfismo sexual y es apreciada por los aficionados a la acuarofilia debido a su polimorfismo y variada colo- ración. Originaria de Trinidad, Barbados, Guyana, Venezuela y Brasil septentrional, esta especie tiene en la actualidad una distribución mundial (Wischnath 1981, Sterba 1983, Halstead et al. 1989, Devezé-Murillo et al. 2004).

El objetivo de este estudio fue determinar la composición, crecimiento e índice de condición de una población de $P$. reticulata, en un estanque en Santo Domingo de Heredia, Costa Rica.

\section{MATERIALES Y MÉTODOS}

Los peces utilizados para este trabajo se obtuvieron de un estanque permanente, localizado en Santo Domingo de Heredia, Costa Rica (9॰59'14"' N y 845'54”'O). En el estanque se midieron mensualmente los siguientes parámetros físico-químicos: temperatura del agua $\left({ }^{\circ} \mathrm{C}\right)$, acidez $(\mathrm{pH})$, dureza $\left(\mathrm{mg} / \mathrm{l}\right.$ de $\left.\mathrm{CaCO}_{3}\right)$ y la concentración en $\mathrm{mg} / \mathrm{l}$ de nitratos, nitritos y oxígeno disuelto (Anónimo 1980). 
Se trabajó con 5086 ejemplares de $P$. reticulata (2 896 machos, 1432 hembras y 758 indeterminados), los cuales fueron recolectados entre setiembre y noviembre de 1998, utilizando una red de mano de nylon rectangular de 60 x $50 \mathrm{~cm}$, de $45 \mathrm{~cm}$ de profundidad y con una luz de malla de $0.05 \mathrm{~cm}$. Se realizaron dos muestreos por mes, con cuatro lances y las muestras fueron preservadas en una solución de formaldehído al $10 \%$.

A cada ejemplar se le midió la longitud total (Lt), longitud estándar (Ls), longitud cefálica $(\mathrm{Lc})$, altura máxima $(\mathrm{Am})( \pm 0.01 \mathrm{~mm})$ y el peso total $(\mathrm{Pt})( \pm 0.01 \mathrm{~g})$. En el caso de las hembras, el peso total se calculó basado en el peso sin la cámara incubatriz ( $\mathrm{P}=\mathrm{Pt}$ - $\mathrm{Pci})$.

Se determinó la relación longitud total-peso según la ecuación $\mathrm{Pt}=\mathrm{a} \mathrm{Lt}^{\mathrm{b}}$ (Ricker 1975). Para determinar la distribución de sexos por tallas y las tallas de la población, los individuos se agruparon arbitrariamente en 14 grupos de tallas: $1: 4.41-7.80 \mathrm{~cm} ; 2: 7.81-11.20 \mathrm{~cm} ; 3: 11.21$ $14.60 \mathrm{~cm} ; 4: 14.61-18.00 \mathrm{~cm} ; 5: 18.01-21.40 \mathrm{~cm}$; 6:21.41-24.80 cm; 7:24.81-28.20 cm; 8: 28.21$31.60 \mathrm{~cm} ; 9: 31.61-35.0 \mathrm{~cm} ; 10: 35.01-$ $38.40 \mathrm{~cm} ; 11: 38.41-41.80 \mathrm{~cm}$; $12: 41.81-45.20$ cm; 13: $45.21-48.60 \mathrm{~cm} ; 14: 48.61-52.00 \mathrm{~cm}$.

El sexo en adultos se determinó por la presencia de gonopodio en machos y por la aleta anal normal en hembras. Se consideró como juveniles a los individuos que no mostraban presencia o desarrollo de gónadas de acuerdo con Constanz (1989) y Reznick (1990).

Se calculó el índice de condición (K) para machos y hembras según la ecuación $\mathrm{K}=\mathrm{a} \mathrm{e}^{\mathrm{b}}$ Lt (Bagenal y Tesch 1978). Se realizaron comparaciones de crecimiento, índice de condición, relación sexual mensual y parámetros morfométricos, entre ambos sexos, mediante la prueba de t-student (Sokal y Rohlf 1981).

Otros aspectos relacionados con la biología de esta especie serán presentados independiente (Urriola et al. 2004).

\section{RESULTADOS}

Los parámetros físico-químicos promedio fueron: temperatura del agua $26.87 \pm 1.29^{\circ} \mathrm{C} ; \mathrm{pH}$ $6.68 \pm 0.44$; dureza $245.01 \pm 25.36 \mathrm{mg} / \mathrm{l} \mathrm{de} \mathrm{Ca-}$ $\mathrm{CO}_{3}$; nitratos $0.63 \pm 0.12 \mathrm{mg} / \mathrm{l}$; nitritos $0.07 \pm$ $0.02 \mathrm{mg} / \mathrm{l}$ y oxígeno disuelto $6.49 \pm 0.69 \mathrm{mg} / \mathrm{l}$.

El análisis estadístico descriptivo, para machos, hembras e indeterminados de $P$. reticulata, (Cuadro 1), reveló que los parámetros morfométricos utilizados muestran diferencias significativas en los promedios, desviaciones estándar y longitudes máximas (t-student $\mathrm{p} \leq 0.05)$. En las longitudes mínimas los valores son semejantes para ambos sexos y el valor

CUADRO 1

Estadística descriptiva por sexos, de una población de P. reticulata en un estanque de Santo Domingo de Heredia, Costa Rica

TABLE 1

Descriptive sex statistics for a population of P. reticulata in a pond in Santo Domingo, Heredia, Costa Rica

\begin{tabular}{|c|c|c|c|c|c|c|c|c|c|c|c|c|}
\hline \multirow[t]{2}{*}{ Parámetros } & \multicolumn{4}{|c|}{$\begin{array}{c}\text { Machos } \\
(\mathrm{n}=2896)\end{array}$} & \multicolumn{4}{|c|}{$\begin{array}{c}\text { Hembras } \\
(\mathrm{n}=1438)\end{array}$} & \multicolumn{4}{|c|}{$\begin{array}{c}\text { Indefinidos } \\
(\mathrm{n}=758)\end{array}$} \\
\hline & Prom. & S & máx. & mín. & Prom & $\mathrm{S}$ & máx & mín. & Prom & S & máx. & mín. \\
\hline $\mathrm{Lt}(\mathrm{mm})$ & 23.50 & 2.24 & 31.70 & 17.80 & 34.43 & 7.56 & 51.50 & 17.80 & 12.27 & 3.41 & 17.80 & 6.90 \\
\hline Ls (mm) & 17.67 & 1.67 & 22.90 & 13.70 & 25.64 & 6.16 & 41.80 & 13.70 & 9.43 & 2.76 & 13.70 & 5.20 \\
\hline $\mathrm{Lc}(\mathrm{mm})$ & 4.79 & 0.39 & 5.50 & 3.90 & 6.49 & 1.13 & 10.20 & 3.90 & 2.87 & 0.75 & 3.90 & 1.70 \\
\hline $\mathrm{Am}(\mathrm{mm})$ & 4.82 & 0.48 & 5.90 & 3.80 & 7.36 & 2.28 & 14.30 & 3.80 & 2.44 & 0.72 & 3.80 & 1.40 \\
\hline Pt $\quad(g)$ & 0.16 & 0.05 & 0.36 & 0.06 & 0.69 & 0.48 & 2.32 & 0.06 & 0.03 & 0.03 & 0.01 & 0.08 \\
\hline
\end{tabular}

Prom. $=$ promedio (average); $\mathrm{S}=$ desviación estándard (standard deviation); min. = mínimo (minimum); max. = máximo (maximum). 
máximo de los indefinidos representó el valor mínimo de los adultos.

La distribución de la población por sexos y por mes (Fig. 1), mostró que el $57 \%$ de los individuos capturados correspondieron a machos, $28 \%$ eran hembras y el $15 \%$ indeterminados. La proporción sexual promedio fue de 1:0.49 (machos:hembras). En términos generales la proporción sexual mensual se mantuvo constante, ya que no se detectaron diferencias estadísticas significativas (t-student $\mathrm{p} \geq 0.005$ ). Por otra parte, se encontró un bajo número de indeterminados $(\mathrm{n}=758)$.

La distribución por tallas y sexos de la población estudiada (Fig. 2) mostró que el porcentaje mayor de individuos (machos y hembras) alcanza entre los 18.01 y $45.20 \mathrm{~mm}$; mientras que los indeterminados presentaron tallas entre los 4.41 y $18.00 \mathrm{~mm}$. El mayor porcentaje se obtuvo para machos entre $21.41 \mathrm{y}$ $24.8 \mathrm{~mm}$.

El índice de condición para hembras se rigió por la ecuación $\mathrm{K}=25.755$ e ${ }^{0.004 \mathrm{Lt}}\left(\mathrm{r}^{2}=\right.$ 0.8925 ), con un promedio de $31.29 \pm 0.55$ y un ámbito entre 28.41 y 32.4 ; mientras que el índice de condición para machos se rigió por la ecuación $\mathrm{K}=26.767 \mathrm{e}^{0.003 \mathrm{Lt}}\left(\mathrm{r}^{2} 0.907\right)$, con un promedio de $28.52 \pm 0.19$ y un ámbito entre 28.18 y 29.05. El análisis estadístico indicó una diferencia significativa entre los valores del índice de condición entre machos y hembras (t-student $\mathrm{p} \leq 0.005$ ), siendo estos mayores en hembras.

Ambos sexos mostraron un comportamiento ascendente en la relación índice de condición-longitud total, manteniéndose sin variaciones durante el período de estudio e iniciándose en los machos a los $17.80 \mathrm{~mm}$ de Lt y en las hembras a los $23.60 \mathrm{~mm}$ de $\mathrm{Lt}$ (Figs. 3 y 4 ).

La relación longitud total-peso para la población, se rigió por la ecuación $\mathrm{Pt}=6 \times 10^{-5} \mathrm{Lt}$ $3 . .3272\left(r^{2}=0.9613\right)$, con un peso promedio de $0.38 \pm 0.41 \mathrm{~g}$ y un ámbito entre $0.01 \mathrm{~g} \mathrm{y} 2.43$ g. Por sexos el peso promedio fue de $0.15 \pm$ $0.05 \mathrm{~g}$ (ámbito = 0.06-0.36 g) para machos, de

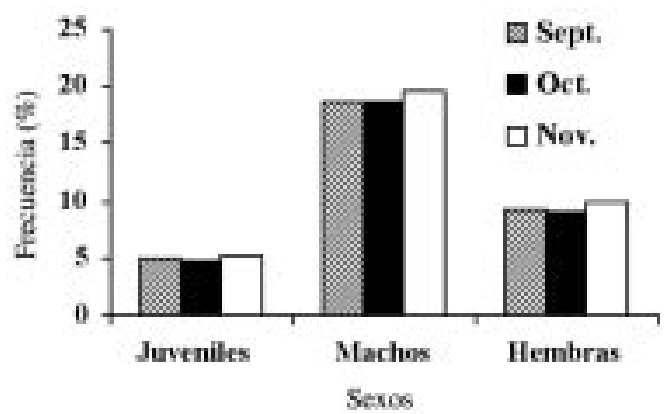

Fig. 1. Distribución de $P$. reticulata por sexos y según mes de muestreo, en un estanque de Santo Domingo de Heredia, Costa Rica.

Fig. 1. P. reticulata sexes distribution by month of collection, in a pond in Santo Domingo, Heredia, Costa Rica.

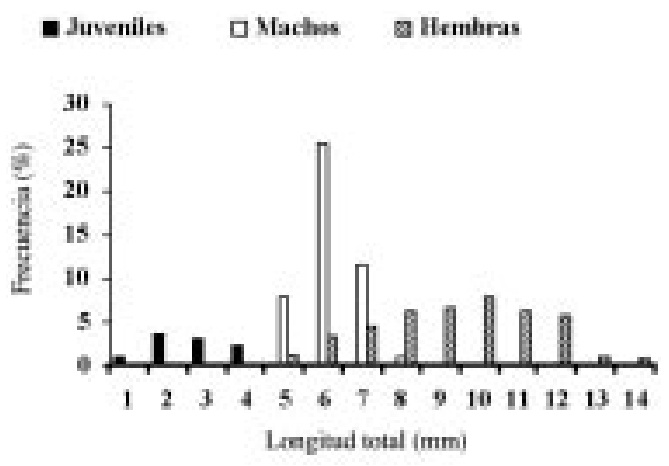

Fig. 2. Distribución de $P$. reticulata por tallas en un estanque de Santo Domingo de Heredia, Costa Rica.

Fig. 2. P. reticulata size group distribution in a pond in Santo Domingo, Heredia, Costa Rica.

$0.69 \pm 0.48$ (ámbito $=0.06-2.32 \mathrm{~g}$ ) para hembras y de $0.03 \pm 0.02 \mathrm{~g}$ (ámbito $=0.01-0.08 \mathrm{~g}$ ) para indeterminados. El análisis estadístico mostró diferencias significativas para la pendiente de las curvas de crecimiento (t-student $\mathrm{p} \leq 0.005$ ), siendo mayores para las hembras. El tipo de crecimiento fue alométrico negativo para machos (2.7138) y alométrico positivo para hembras (3.3554).

Las hembras y machos alcanzaron la madurez sexual y son reproductivamente activos en tallas superiores a los 23.6 y $20.00 \mathrm{~mm}$ de Lt respectivamente. 


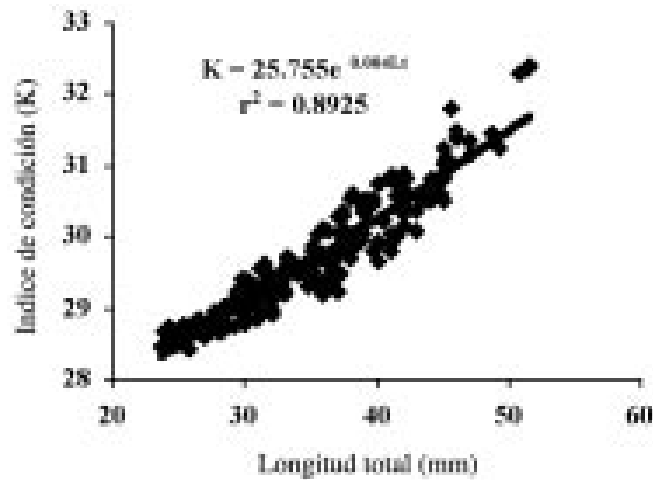

Fig. 3. Relación entre índice de condición y longitud total para hembras de $P$. reticulata en un estanque en Santo Domingo de Heredia, Costa Rica.

Fig. 3. Correlation of total length and conditional index for females of $P$. reticulata in a pond in Santo Domingo, Heredia, Costa Rica.

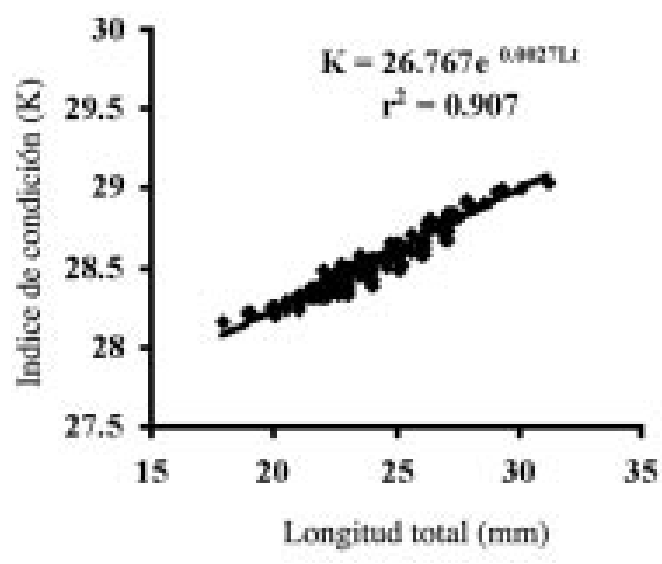

Fig. 4. Relación entre índice de condición y longitud total para machos de $P$. reticulata en un estanque en Santo Domingo de Heredia, Costa Rica.

Fig. 4. Correlation of total length and conditional index, for males of $P$. reticulata in a pond in Santo Domingo, Heredia, Costa Rica.

\section{DISCUSIÓN}

Los parámetros físico-químicos se encuentran dentro de los ámbitos señalados por Jacobs (1971), Gannon (1974), Sterba (1983), Halstead et al. (1989) y Gómez-Marquez et al. (1999) para poecílidos.
Esta especie presentó un marcado dimorfismo sexual en tamaño, lo cual puede ser atribuido a que en la familia Poeciliidae las hembras demoran más en madurar que los machos y estos últimos son reproductivamente precoces; lo que coincide con lo informado por Jacobs (1971), Barus et al. (1987), Farr (1989), Greenfield (1990) y Vargas y de Sostoa (1996).

La proporción sexual favoreció a los machos sobre las hembras (1:0.49), lo cual difiere de lo informado por Reza y Díaz (1994) para Heterandria bimaculata (1:2.3), por Contreras-McBeath y Ramírez (1996) para Poeciliopsis gracilis (1:4.5) y por Vargas y de Sostoa (1996) para Gambusia holbrooki (1:4).

La longitud total máxima encontrada para las hembras de $P$. reticulata fue de $51.5 \mathrm{~mm}$, semejante a la informada para gupis finos por Halstead et al. (1989), para Poecilia teresae en Belice por Greenfield (1990) y menor que la informada para gupis por Sterba (1983) (60 $\mathrm{mm}$ ). La longitud máxima encontrada para los machos de $P$. reticulata fue de $31.5 \mathrm{~mm}$, semejante a la informada por Sterba (1983) y por Halstead et al. (1989) para gupis y menor que la informada por Greenfield (1990) (47.5 mm) para $P$. teresae.

El bajo número de indeterminados se puede atribuir a la presencia de Bufo marinus, $R a$ na taylori, Ceryle torcuata, Pitangua sulfuratus, Tiranus melancholicus, Quiscalus mexicanus, Aninga aninga, ya que se les observó alimentándose de peces principalmente en la riberas del estanque, coincidiendo con lo informado por Rood y Reiznick (1997).

El índice de condición es evidente a partir de las tallas $17.8 \mathrm{~mm}$ en machos y $23.6 \mathrm{~mm}$ en hembras y se incrementa en forma más evidente a medida que aumenta la longitud total, lo que refleja la influencia positiva de las condiciones ambientales en el crecimiento de los individuos (Snelson 1989, Reznick y Miles 1989, Sparre 1992)

La relación longitud-peso para machos y hembras, sugiere un alto grado de dimorfismo sexual, dado los valores diferentes para el 
factor de crecimiento (2.7138 y 3.3554) para machos y hembras, respectivamente), lo que indica que los valores altos de alometría tienden hacia una forma más esférica del cuerpo, de manera que los machos son más delgados y alargados, lo que coincide con lo informado por Jacobs (1971) y Sterba (1983), Safran (1992) y Gómez-Márquez et al. (1999).

La talla a la cual $P$. reticulata inicia su etapa reproductiva es menor en machos que en hembras, lo cual es atribuible a que la tasa de crecimiento de éstos es mayor, como respuesta a la competencia en los eventos reproductivos, coincidiendo con Sumner et al. (1994) y Gómez-Márquez et al. (1999).

\section{RESUMEN}

Se determinó la composición, crecimiento e índice de condición de una población de Poecilia reticulata, en un estanque en Santo Domingo de Heredia, Costa Rica, entre setiembre y noviembre de 1998. La proporción sexual promedio fue de 1 macho: 0.49 hembras. Las hembras presentaron una longitud promedio total de $34.43 \pm$ $7.26 \mathrm{~mm}$ y un peso total promedio de $0.69 \pm 0.48 \mathrm{~g}$, mientras que en los machos la longitud total promedio fue de $23.46 \pm 2.24 \mathrm{~mm}$ y el peso total promedio de $0.16 \pm 0.05$ g. En los indeterminados la longitud total promedio fue $12.27 \pm 3.41 \mathrm{~mm}$ y un peso total promedio de $0.026 \pm$ $0.027 \mathrm{~g}$. La relación longitud total-peso total para toda la población se rigió por la ecuación $\mathrm{P}=6 \times 10^{-5} \mathrm{Lt}^{3.3272}\left(\mathrm{r}^{2}\right.$ $=0.9613)$. El índice de condición para hembras se rigió por la ecuación $\mathrm{K}=25.755 \mathrm{e}^{0.004 \mathrm{Lt}}\left(\mathrm{r}^{2}=0.8925\right)$, con un promedio de $31.29 \pm 0.55$ y para machos por la ecuación $\mathrm{K}=$ $26.767 \mathrm{e}^{0.003 \mathrm{Lt}}\left(\mathrm{r}^{2}=0.907\right)$, con un promedio de $28.52 \pm 0.19$. La talla al momento de la primera reproducción para machos fue de $20.00 \mathrm{~mm}$ de Lt y para las hembras de $23.5 \mathrm{~mm}$.

\section{REFERENCIAS}

Anónimo. 1980. Standard methods for the examination of water and wastewater. American Public. Health Association. Washington, D.C., Estados Unidos. 874 p.

Bagenal,T.B. \& F.W. Tesch. 1978. Age and growth methods for assessment of fish production in fresh waters. IBP handbook $\mathrm{N}^{\circ}$ 3. Blackwell. Oxford. pp. 101-136.

Barus, V., J. Libosvarsky \& A. Coy Otero. 1987. Poeciliids in central Cuba. Folia Zool. 36: 57-62.
Bussing, W.A. 1998. Peces de las aguas continentales de Costa Rica. Universidad de Costa Rica, San José, Costa Rica. 468 p.

Constantz, G.D. 1989. Reproductive Biology of Poeciliid Fishes, pp. 33-50. In G.K Meffe. \& F.F. Snelson Jr (eds.). Ecology and Evolution of Livebearing fishes (Poeciliidae), Englewood Cliffs, Nueva Jersey, Estados Unidos.

Contreras-McBeath, T. \& H.E. Ramírez. 1996. Some aspects of the reproductive strategy of Poeciliopsis gracilis (Osteichthyes:Poeciliidae) in the Cuautla river, Morelos, México. J. Freshw. Ecol. 11: 327-338.

Devezé-Murillo, P., J.L. Reta Mendiola, B. Sánchez Luna. 2004. Desarrollo tecnológico para la producción de Poecilia reticulata (Pisces: Poeciliidae) en cuerpos de agua tropicales de Veracruz, México. Rev. Biol. Trop. 52: en prensa.

Farr, A J. 1989. Sexual selection and secondary sexual differentiation in poeciliids: Determinants of male mating success and the evolution of female choice, pp. 91-123. In G.F. Meffe \& F.F. Snelson Jr. (eds.). Ecology and Evolution of Livebearing fishes (Poeciliidae), Englewood Cliffs, Nueva Jersey, Estados Unidos.

Gannon, R. 1974. Starting right with tropical fish. T.F.H., Nueva York, Estados Unidos. 64 p.

Gómez-Marquez, J.L., J.L. Guzmán-Santiago \& A. Olvera-Soto. 1990. Reproducción y crecimiento de Heterandria bimaculata (Cyprynodontiformes: Poeciliidae) en la laguna "El Rodeo", Morelos, México. Rev. Biol. Trop. 47: 581-592.

Greenfield, D. 1990. Poecilia teresae, a new species of poeciliid fish from Belize, Central America. Copeia 2: $449-454$.

Halstead, B., B. Landa \& G.F. Sandstrom. 1989. A golden guide tropical fish. Golden, Wisconsin, Estados Unidos. $160 \mathrm{p}$.

Jacobs, K. 1971. Livebearing Aquarium Fishes. Studio, Leipzig, Alemania. 459 p.

Lody, E. 1978. Chromosome complement of the guppy, Poecilia reticulata Peters. (Pisces: Osteichthyes). Caryología 4: 476-479.

Reza, U.B.S. \& P.E. Díaz. 1994. Algunos aspectos de la biología reproductiva de Heterandria bimaculata (Poeciliidae). Resúmenes IV Congreso Nac. Ictiol., Morelia, Michoacán, México. pp. 57-58.

Reznick, D. 1981. The impact of life history evolution in Trinidadian guppy (P. reticulata). Evolution 36: 160-177. 
Reznick, D. 1990. Plasticity in age and size at maturity in male guppies (Poecilia reticulata): An experimental evaluation of alternative models of development. J. Evol. Biol. 3: 185-203.

Reznick, N.D. \& D.B. Miles. 1989. A review of life history patterns in poeciliid fishes, pp. 125-148. In G.F. Meffe \& F.F. Snelson Jr. (eds.). Ecology and Evolution of Livebearing fishes (Poeciliidae), Englewood Cliffs, Nueva Jersey, Estados Unidos.

Ricker, W.E. 1975. Handbook of computations for biological statistics of the fish population. Bull. Fish. Res. Bd. Can. 191: 382.

Rood, F.H. \& D.N. Reznick. 1997. Variation in the demography of guppy populations: the importance of predation and life histories. Ecology 78: 405-418.

Safran, P. 1992. Theoretical analysis of the weight-length relationship in fish juveniles. Mar. Biol. 112: 545-551.

Sparre, P. 1992. Introducción a la evaluación de recursos pesqueros tropicales. Manual FAO. Chile. 245 p.

Snelson, F.F.Jr. 1989. Social and environmental control of life history traits in poeciliids, pp. 149-161. In G.F. Meffe \& F.F. Snelson Jr. (eds.). Ecology and Evolution of Livebearing fishes (Poeciliidae), Englewood Cliffs, Nueva Jersey, Estados Unidos.
Sokal, R.R. \& F.J. Rohlf. 1981. Biometry. Freeman, Nueva York, Estados Unidos. 859 p.

Sterba, G. 1983. The Aquarium Encyclopedia. Mills, Nueva York, Estados Unidos. 608 p.

Sumner, I.T., J. Travis \& C.D. Johnson. 1994. Methods of female fertility advertisement and variation among males in responsiveness in the sailfin molly (Poecilia latipinna). Copeia 1: 27-34.

Urriola Hernández, M., J. Cabrera Peña \& M. Protti. Fecundidad, fertilidad e índice gonadosomático de Poecilia reticulata (Pisces: Poeciliidae) en un estanque en Santo Domingo, Heredia, Costa Rica. Rev. Biol. Trop. 52: en prensa.

Vargas V., M. \& J.V. Vargas C. 2003. Male and mosquito larvae survey at the Arenal-Tempisque irrigation project, Guanacaste, Costa Rica. Rev. Biol. Trop. 51: 759-762.

Vargas, M.J. \& A. de Sostoa. 1996. Life history of Gambusia holbrooki (Pisces, Poeciliidae) in the Ebro delta (NE Iberian peninsula). Hydrobiology 341: 215-224.

Wischnath, L. 1981. Los guppies salvajes de México. Vida Acua. 34: 252-312. 